\title{
Carnets
}

Revue électronique d'études françaises de l'APEF

Deuxième série - 19 | 2020

Petite fabrique d'interprètes

\section{L'enchantement de la lecture chez Alphonse Daudet ou la champignonnière de grands hommes}

\section{Lúcia Bandeira}

\section{(2) OpenEdition}

1 Journals

Édition électronique

URL : http://journals.openedition.org/carnets/11916

DOI : 10.4000/carnets. 11916

ISSN : 1646-7698

Éditeur

APEF

\section{Référence électronique}

Lúcia Bandeira, «L'enchantement de la lecture chez Alphonse Daudet ou la champignonnière de grands hommes », Carnets [En ligne], Deuxième série - 19 | 2020, mis en ligne le 31 mai 2020, consulté le 23 décembre 2020. URL : http://journals.openedition.org/carnets/11916 ; DOI : https://doi.org/ 10.4000/carnets. 11916

Ce document a été généré automatiquement le 23 décembre 2020.

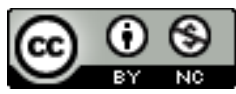

Carnets est mis à disposition selon les termes de la licence Creative Commons - Atribution - Pas d'utilisation commerciale 4.0 International. 


\title{
L'enchantement de la lecture chez Alphonse Daudet ou la champignonnière de grands hommes
}

\author{
Lúcia Bandeira
}

1 La lecture, le livre, l'objet écrit et les écrivains tiennent une place prépondérante aussi bien dans la vie de Daudet que dans la vie des personnages qu'il consacre dans ses œuvres. La lecture s'imprime dans la vie et s'enchaîne sur les mailles de l'écriture. Âme nostalgique, Daudet aimait noter sur le papier toute sorte de souvenirs, d'impressions du moment. Entre Nîmes où il est né, Lyon où il s'est vu planté et Paris où il se replanta, l'auteur des Lettres de mon moulin absorbait toute sorte de sensations que seul l'acte d'écrire sur le papier assouvissait.

2 L'écriture était devenue un mode de vie, et Daudet cherchait dans les mots le reflet de l'âme. Adeline Reynaud, sa mère, avait bien compris à quel point la lecture pouvait influencer une âme sensible comme celle d'Alphonse. Elle-même se plaisait à lire, en cachette, des romans sentimentaux. À son commerce de la soie, elle volait des heures pour s'emporter dans le rêve que la lecture lui procurait. Elle avait ainsi transmis à son fils non seulement le goût précoce de la lecture, mais la soumission même des lectures à la cadence de la vie. Le jeune Alphonse se mettait donc à vivre comme Robinson Crusoé; il rêvait de vivre sur une île, en rapport avec la nature, comme «un Robinson ».

3 Il a appris à lire dans une petite école, à Besouce, où il parlait en patois malgré l'interdiction formelle de ses parents. Plus tard, le Midi et le patois provençal que l'éloignement avait sublimés sont devenus une source d'inspiration inépuisable qui lui ont permis de se rapprocher de ses racines et d'admirer tous les écrivains provençaux, s'étant lui-même adonné à l'écriture en provençal. Il lisait fiévreusement les poètes provençaux dont Mistral était le plus noble représentant. L'amitié Mistral-Daudet reposait sur l'amour porté à la langue provençale et à la poésie. La correspondance 
échangée tout au long de leurs vies atteste l'intérêt porté à la littérature en général, et à celle du Midi, en particulier à la langue et à sa divulgation.

4 À Lyon, la meilleure école que Daudet trouve à sa formation de poète est l'école buissonnière. Dans son petit canot, il parcourt le fleuve dont les remous et les reflets l'enchantent. Le soir, il lit avec son frère toute sorte de romans, en cachette, bien entendu ! À Paris, Alphonse Daudet mène une vie de bohème entre la vie sociale et la vie amoureuse. C'est son frère Ernest qui l'introduit dans les salons littéraires et les cafés journalistiques, en quête de relations avec des écrivains célèbres.

En 1865, Alphonse Daudet rencontre au théâtre de la Comédie Française, lors d'une représentation d'une pièce des frères Goncourt, Julia Allard, qu'il épouse en 1867. Femme intelligente et attachée à la littérature, elle sera la première lectrice de son mari. Julia est une femme distinguée, qui motive Daudet à l'écriture, corrige ses travaux, voire ajoute à sa guise. Daudet mène dorénavant une vie rangée, avec un rythme de travail suivi. Il est difficile de juger la participation active de Julia dans la production littéraire de Daudet. Bien qu'il soit possible de constater sur certains manuscrits les corrections apportées par Julia, il est difficile, dans cette collaboration littéraire, de discerner la nature réelle de l'apport de l'un et de l'autre. Edmond de Goncourt reconnaît, à Julia, des qualités exquises de lectrice. Il lui rend hommage dans la revue Art, où il affirme n'avoir jamais rencontré quelqu'un qui ait aussi bien lu qu'elle, mais aussi dans son Journal, où la référence à Madame Daudet est récurrente.

6 L'activité littéraire de Daudet est intense. Il écrit / travaille pendant de longues heures dans les cabinets de travail élus à cet effet. Au travail dans le recueillement, s'oppose l'agitation du salon littéraire. Daudet a connu l'agitation du salon du Duc de Morny. Il fréquente les salons des auteurs naturalistes, dîne régulièrement chez Zola aux soirées de Médan; le dimanche, il fréquente le grenier de Goncourt à Auteuil. Lui-même reçoit, chez lui, ces amis des réunions littéraires et artistiques. Les salons littéraires parisiens permettaient à tous et à chacun de se «mettre à la page ». Avec l'arrivée du chemin de fer, il devient plus aisé de s'éloigner de Paris ou de s'y rendre, et nous assistons à la construction de demeures bourgeoises à la campagne. Avec ces atouts s'installe la mode du salon littéraire de villégiature pendant les mois d'été.

7 Le salon Daudet dans le quartier de Champrosay, à Draveil, gagne une énorme réputation et, tous les jeudis, des journalistes, des écrivains, des peintres se réunissent autour d'une table pour manger et discuter. Les jeudis de Champrosay, comme dans tout autre salon de l'époque, permettaient aussi des rencontres de gens liés pour des raisons politiques et/ou littéraires. Les ennemis ont parfois besoin de se rencontrer pour se contredire, s'attaquer, se situer! Edmond de Goncourt écrit dans son Journal, le 10 avril 1886: "Nous sommes dans le salon, je dis deux ou trois choses qui sont contredites par Zola, je me sens devenir nerveux, et me voici emballé avec Zola dans une discussion sur l'esprit ». (Goncourt, 1956, vol. II : 1239-1240)

8 Toutefois, ces discussions ne dissuadent pas les auteurs de fréquenter le salon. En effet, sept ans plus tard, le différend Zola-Goncourt n'est pas résolu. Edmond de Goncourt écrit le 4 août 1893 que tous les six mois, Zola avait une curiosité à tâter le pouls à Daudet et à lui-même, une curiosité à savoir où ils en étaient physiquement et d'un point de vue cérébral. Cela rendait tous les présents nerveux.

9 Les auteurs ont donc besoin d'échanger leurs idées et leurs impressions dans un cadre stimulant leur permettant de garder les liens avec le milieu littéraire, artistique, journalistique et politique. La fréquentation du cercle littéraire était indispensable. 
Chez Daudet, beaucoup d'auteurs consacrés se côtoient: Leconte de Lisle, Goncourt, Anatole France, José Maria de Hérédia, Marcel Proust, Octave Mirbeau, entre autres. Le plus intime de ces hôtes est sans doute Edmond de Goncourt qui trouve chez les Daudet, surtout après la mort de son frère, Jules, une ambiance réconfortante. Edmond avait sa chambre chez Daudet, et c'est dans cette maison qu'il s'éteint le 16 juillet 1896.

"Salon littéraire ", " cercle ", " cénacle ", « société littéraire " (fondation des frères Goncourt), "foyer littéraire », sont des expressions qui soutiennent la portée de la lecture en cette deuxième moitié du XIXe siècle. Le rapport de Daudet à la lecture ne sied pas sur un rapport institutionnel ou uniquement professionnel. Une grande réflexion sur les mots, sur certains écrivains, sur la philosophie, sur la lecture se dégage des œuvres et des notes prises au hasard du moment sous l'inspiration. De la lecture des notes, revues et organisées par Julia, on peut dresser une liste d'auteurs cités par Daudet, lesquels semblent entrer dans une danse de textes et d'idées: La Fontaine, Sénèque, Sainte-Beuve, Sand, Tourgueniev, Balzac, Horace, Champfleury, Hugo, Goncourt, Banville, Dostoïevski, Stendhal, Cottin, Poe, Goethe, Baudelaire, Musset, Verlaine, Montaigne, Diderot, Boileau, Voltaire, Taine, Constant...

11 Les sujets des notes de Daudet sont diversifiés, mais il n'est pas difficile de les classer. On y trouve des réflexions sur la condition humaine, surtout la vue de scènes émouvantes, des descriptions de lieux, surtout d'éléments naturels : l'eau, la terre (la nature), des citations d'auteurs, des commentaires sur certains auteurs, des commentaires sur la philosophie et sur les philosophes, sur le style littéraire et sur la lecture.

12 Son attachement à la lecture, suivi d'un travail d'écriture de longue haleine et de lecture de toutes les nouveautés du domaine littéraire, a façonné son idée de style. À titre d'exemple, nous présentons une citation sur le style, plus précisément sur certaines figures de styles qui à force de recours excessifs deviennent usées et épuisent l'effet littéraire. À croire, selon Daudet, que les clichés fatiguent l'œuvre :

Quel ennui profond doivent éprouver les épithètes qui vivent depuis des siècles avec les mêmes substantifs. Les mauvais écrivains ne veulent pas comprendre cela : ils croient que le divorce des mots n'est pas permis. Il y a des gens qui ne rougissent pas d'écrire : des arbres séculaires, des accents mélodieux. Séculaire n'est pas laid, mettez-le avec un autre substantif: mousses séculaires, jardins séculaires, etc.; voyez, il fait bon ménage. Bref, l'épithète doit être la maîtresse du substantif, jamais sa femme légitime. Entre les mots il faut des liaisons passagères, mais pas de mariage éternel. C'est ce qui différencie l'écrivain original des autres. (Daudet, $1929: 2$ ).

13 Daudet semble entamer vivement un traité de stylistique, et se présenter comme un critique littéraire, un écrivain chevronné et même comme conseiller, didacticien, lorsqu'il interpelle son interlocuteur, comme le prouve l'impératif: "mettez-le", «voyez ». Au-delà de ces réflexions, Daudet constate que le lieu et les circonstances où la lecture s'opère déterminent fortement la réception de l'œuvre et influence la portée de cette même lecture :

Comme tout se tient! par quel fil mystérieux nos âmes sont liées aux choses : une lecture faite dans un coin de la forêt et en voilà pour toute la vie. Chaque fois que vous penserez à la forêt, vous reverrez le livre ; chaque fois que vous relirez le livre, vous reverrez la forêt. Pour moi qui vis beaucoup aux champs, il y a des titres d'ouvrages, des noms d'auteurs qui m'arrivent dans un développement de parfums, de sons, de silences, de fonds d'allées. Je ne sais plus quelle nouvelle de Tourgueniev 
est restée dans mon souvenir sous la forme d'un petit flot de bruyère rose, un peu fanée déjà par l'automne (idem : 21). l'absolue absence d'algèbre et de géométrie, mon année de philosophie tronquée et sans direction. De là ma répugnance aux idées générales, aux abstractions, l'impossibilité où je me trouve d'avoir une formule quelconque sur toute question philosophique » (idem : 34 ). 
20 L'enchantement de la lecture chez Alphonse Daudet lui parvient aussi par le plaisir de l'écriture, de l'association des mots et des images, de la vue de la beauté et de la bonté qu'il inscrit dans sa façon d'être, dans sa façon de vivre et d'écrire. En tant que bâtisseur d'émotions, sensible et perméable à tous les sens, il désire depuis sa tendre enfance écrire toutes les sensations provoquées par la vie. Pas étonnant dès lors de retrouver dans la plupart de ses ouvrages des personnages attachés à la lecture et à l'écriture, comme lui-même l'a été sa vie durant.

21 L'homme Alphonse Daudet est le témoin de l'expansion envahissante du monde du livre, qu'il consigne dans toute sa production littéraire, ainsi que dans son modus vivendi. Daudet ne dénonce pas l'ignorance de façon effusive. Par contre, il insiste sur la lecture en tant que moyen de formation et d'élévation de l'esprit de l'individu. Jack, par exemple, désespère dans son acheminement vers la compréhension de certains textes ; toutefois il avoue comprendre l'horreur du travail à l'usine qu'il associe à L'Enfer de Dante.

Ce souci de formation de l'individu par la lecture, il l'a lui-même expérimenté puisqu'il se disait un grand « liseur », mais a poussé plus loin cette expérience en consignant la lecture dans l'objet littéraire, en postulant de la sorte l'importance vitale de la lecture. En ce sens, Daudet témoigne de la relation fusionnelle entre l'écriture, la lecture et la vie.

23 En tant qu'auteur, sa production littéraire rapporte le contexte culturel de la deuxième moitié du XIXe siècle, ce qui atteste son engagement dans le courant intellectuel de cette époque qui préconisait l'écriture au service de l'étude des mœurs. Les titres de journaux, de romans et des noms d'auteurs peuplent sa production littéraire. La possibilité de dresser une liste des auteurs cités et convoqués par Daudet, nous ramène à la métaphore de Jean Bruller, dit Vercors, dans Le silence de la mer retenue dans le discours de Werner Von Ebrennac, lequel évoque la difficulté à saisir un auteur français qui puisse être représentatif de la littérature française. Il est évident pouvoir affirmer que Shakespeare représente les Anglais ; Dante, les Italiens ; Cervantès, les Espagnols ; Goethe, les Allemands. Mais qui citer pour représenter la France : Molière? Racine? Hugo ? Voltaire? Rabelais ? Ou quel autre? Werner Von Ebrennac les compare à une foule qui se presse à l'entrée d'un théâtre, sans que l'on sache qui faire entrer en premier. C'est tout à fait cette foule à l'entrée du théâtre que l'on trouve dans l'œuvre daudétienne. Daudet exprime, signale, se porte à témoin de la richesse du patrimoine littéraire français, voire étranger.

24 Au-delà de la fresque littéraire qui jaillit de son œuvre, Daudet peint également la démarche de l'auteur auprès des éditeurs, ceux que Goncourt appelle « les marchands de copie ", mais s'attache aussi à la manipulation de l'auteur aux mains du "seigneur public ", avide de nouveautés; un public critique et exigeant auxquels les éditeurs se plient en publiant à la mesure des bourses de chaque couche sociale des livres reliés pour les uns, des feuilletons pour les autres, des journaux pour tout un chacun. Toute la peinture des mœurs et du monde du papier du XIXe siècle en effervescence est fixée transversalement dans les écrits de Daudet.

25 La production littéraire de ses débuts de carrière semble assez ancrée sur des modèles, sur le soutien des éditeurs et des amis de lettres avec qui il partageait ses projets de livre, ou avec qui il inventait des intrigues pour un nouveau livre, tout en parlant. Il aimait à expérimenter sur les autres, tout en parlant. Il testait, dans les conversations avec son entourage, ses projets de travail écrit. Cette caractéristique de l'homme 
qu'était Daudet atteste sa propension à l'oral. Mme Bost, actuelle propriétaire de la maison Daudet à Draveil affirmait, lors d'un entretien dans le parc, que Daudet était avant tout un conteur, un auteur de l'oral. Goncourt, lui aurait confié que Daudet était timide, d'un style de petite qualité, mais un faiseur de comédies avec une énorme facilité de conversation et d'improvisation. Toutefois Daudet aura su, à force de travailler, affirmer son autonomie, sa lucidité d'esprit et sa réflexion critique, même quand la maladie s'était installée, en confrontant dans la dernière lettre de Charlexis, de La Petite Paroisse, (un des derniers romans de Daudet) la question philosophique sur la dialectique écrivain / société :

Oh! non, il n'y a pas de flamme dans les yeux de notre génération, n'est-ce pas, Vallongue? Nous ne brûlons pas plus pour l'amour que pour la patrie. À qui la faute ? Vous, mon philosophe, penseur, piocheur, dévoreur de bouquins, c'est dans les brouillards de la métaphysique allemande (...) ; vous accusez les livres de vous avoir instruit et desséché trop tôt. Mais alors, nous autres, les cancres, nous qui ne lisons pas, nous aurions dû le garder, ce foyer d'honnêtes croyances, et c'est tout le contraire. Probablement, les lourds bouquins qui vous ont désenchanté, il n'est pas besoin de les ouvrir pour les connaître; les désespérantes idées, qu'ils contenaient comme en germe, se sont formulées et dispersées, et nous les respirons avec l'air et la vie, nous les absorbons par tous les pores (...). Voilà pourquoi, nous tous du dernier bateau, celui de la conquête, ignorants comme moi ou savants comme vous, nous sommes tous frappés d'ennui et d'épuisement, vaincus avant l'action, tous des âmes d'anarchistes à qui le courage du geste a manqué. (Daudet, 1991 : 172).

Tout le parcours littéraire de Daudet et de son époque tient dans cet extrait : tous les écrivains essaient de se frayer un chemin dans la lutte pour la vie, c'est le cadre d'« Une champignonnière de grands hommes", où tous rêvent de participer au bien être de l'humanité, mais le courage de l'action leur a manqué. Les théories ont été vaines, le mal de vivre s'installe. Le roman social toucherait-il à sa fin? Les principes du Naturalisme ne sont-ils pas mis en cause? Et ceux du symbolisme ne sont-ils pas justifiés d'office?

Daudet traite des sujets tels que la formation intégrale de l'individu par l'étude consciente et suivie des auteurs, mais il aborde de même le thème de l'écriture comme moyen de fixer l'Histoire des hommes, et l'histoire de chaque homme. S'il est vrai que ces sujets ne sont pas élevés au niveau de l'idéologie, on ne peut nier la présence de livres, de bibliothèques, de journaux, de scènes de lecture dans toute sa production littéraire. Il s'attache aussi, bien que subtilement, à l'effet de la lecture sur quelques personnages qui peuplent sa production littéraire, tels que Le Petit chose, Jack, Tartarin, Delobelle, le Dr. Rivals, Cécile, Lydie, Charlexis, Didier, entre autres.

En tant qu'auteur, Daudet consigne des noms d'écrivains ayant connu la notoriété, d'autres à qui la chance a joué des tours comme à Delvau, mort avant d'avoir lu son livre qui venait d'être publié : «Le dernier livre » que nous pouvons lire dans Lettres de mon moulin. Du point de vue thématique, on affirmer que l'œuvre de Daudet contribue à l'étude du milieu culturel, littéraire et social de la seconde moitié du XIXe siècle, ainsi qu'à l'étude du foisonnement artistique, politique et intellectuel. Toute son époque y tient !

29 Ainsi, donc, depuis le temps du Petit Chose, misérable et miséreux, sans argent pour s'acheter des livres qu'il ne lisait que chez les libraires ou parce qu'on les lui prêtait, jusqu'au temps de M. Daudet, homme de lettres, possédant une bibliothèque bien garnie, c'est bien par la lecture que Daudet a gagné sa vie. Mais quelle conception de lecture(s) peut-on dégager de l'œuvre de Daudet ? Daudet était un homme de lettres. J.- 
Henri Bornecque le définit Daudet comme "un romantique de cœur, un classique d'esprit, si l'on peut arbitrairement séparer des tendances qui se font équilibre " (Bornecque, 1951 : 110).

On peut cerner le parcours de Daudet vers les classiques, les romantiques, comme Musset qu'il cite dans "L'arrivée ", Trente ans de Paris, et dans un article au Journal Officiel du 14 juin 1875 où il avoue son admiration pour le poète. Alors qu'il évoque ses poètes préférés, Daudet se consacre à la poésie, puis au conte où il raconte des histoires courtes et significatives. C'est le temps des Lettres de mon moulin, des Contes du lundi, des Lettres à un absent. Par la suite, Daudet écrira des œuvres romanesques qui représentent l'aboutissement d'un travail de recherche de/sur soi et des/sur les autres.

Aussi remarque-t-on que la lecture (et l'écriture) était une profession, mais qu'elle lui donnait du plaisir, de l'enchantement ; elle lui ouvrait le chemin de la magie et du rêve, bien que la tâche de la lecture lui était rendue difficile dans certains moments de la vie. Que de joies éprouvées et exprimées par la lecture de poèmes avec ses amis de la Provence, de romans! Le livre est parfois personnifié ; il le caresse, en prend soin, le sublime. Daudet ne peut se départir de cette conception de lecture liée à l'essence même de la vie.

Le thème de la lecture est présent dans sa production littéraire par des personnages depuis les premières publications où, aussitôt, les Lettres de mon moulin retiennent notre attention. La lettre, bien que fictive, implique l'acte de lecture. Dans ce cadre Provençal, le narrateur y cherche la bibliothèque des cigales. Le petit Alsacien de La dernière classe n'est-il pas dans un contexte d'apprentissage de la lecture? Il lui faudra même apprendre à lire en allemand! Et Bixiou, représentant de la misère du monde des lettres, n'est-il pas un grand journaliste et lecteur de journaux? Devenu aveugle, la lecture lui manque. Même sa femme ne trouve pas le temps de lui faire la lecture !

Le Petit Chose ne cherchait-il pas à mettre en action sa lecture de Robinson Crusoé ? Tartarin, lui aussi, voulait mettre ses lectures en action ; il se donne le rôle des héros. Il est tantôt D. Quichotte, tantôt Sancho Panza. Il reconnaît que certaines lectures lui ont façonné l'esprit, mais qu'elles ne correspondent pas à la réalité. Il va connaitre la désillusion en Algérie, car ce qu'il y voit ne correspond pas à ce qu'il a lu dans Mille et une nuits.

Dans Fromont jeune et Risler aîné, Delobelle relit et cite les auteurs dramatiques; les employées de Melle Mire qui enfilent des perlent, lisent des feuilletons qui traînent dans les tiroirs. Frantz, qui a toujours beaucoup lu, se trouve à la gare de Lyon, où il attend Sidonie. Pour tromper la fatigue, il lit les titres dans la boutique de livres. Jack, suit tout un programme de formation et d'éducation qui lui est dressé à partir de la lecture orientée de quelques œuvres.

Le cabinet de lecture de Mme Lévêque est fréquenté par des filles des usines qui sont affamées de lectures romanesques. Certaines laissent des notes dans les marges des pages, ce qui souligne leur intérêt pour la lecture. Ida, la mère de Jack a lu des romans, mais elle ne questionne jamais ; n'intègre pas les lectures dans son élévation d'esprit. Le Dr. Rivals et sa petite fille représentent les lecteurs avertis, pédagogues, critiques. Charlexis, lui, a beaucoup lu, mais sa conduite est pourtant méprisable parce qu'il révèle un comportement d'aventurier. Il s'interroge toutefois sur le rôle des grands auteurs dans la société. Lydie est devenue une liseuse de romans étrangers, ce qui 
atteste son évolution intellectuelle et la correction de sa posture. La lecture de ces livres est un indice de sa volonté de vivre dans l'honneur.

À ces noms de lecteurs dans ses romans, viennent s'ajouter ceux que nous trouvons dans la production dramatique, dans laquelle livres et journaux cohabitent avec tous les autres éléments du décor. Il en va de même dans la production poétique. Le livre et la lecture sont omniprésents et traversent tous les genres littéraires.

Daudet a vécu le défi du mot, la difficulté à écrire, la difficulté de l'édition, le désarroi du manque de temps pour tout consigner sur le papier. L'homme qui voulait être un " marchand de rêves » a vécu l'enchantement de la lecture et le plaisir à connaître les hommes de lettres. Il est le maître qui croit en la formation de l'individu par le biais du travail sur la lecture et sur la formation en littérature. L'individu est à même de conquérir sa liberté (ou de la perdre !) par l'engagement dans le monde du livre. Son œuvre, à (re)découvrir, témoigne un foisonnement de sujets autour du monde artistique et littéraire dont la source est la provocation du mot et la responsabilité de le dire.

\section{BIBLIOGRAPHIE}

BÉRAUD, Henri (1930). CEuvres complètes illustrées, Édition Ne Varietur, précédé de « Retour sentimental vers Alphonse Daudet » par Henri Béraud ; Paris : Librairie de France.

BORNECQUE, Jacques-Henry (1979). Histoire d'une amitié. Correspondance entre Alphonse Daudet et Frédéric Mistral (1860-1897). Paris : Julliard.

BORNECQUE, Jacques-Henry (1951). Les années d'apprentissage. Paris : Librairie Bizet.

DAUDET, Alphonse (1929). Notes sur la Vie. Paris : Librairie de France.

DAUDET, Alphonse (1991). La Petite Paroisse. Paris : Les Feuillantines.

DAUDET, Alphonse (1990). Jack. Paris: Gallimard.

DAUDET, Léon (1940). Quand vivait mon père (souvenirs inédits). Paris : Grasset.

GONCOURT, Edmond (1956). Journal - Mémoires de la vie littéraire. Paris : Robert Laffont.

RIPOLL, Roger (1986). Alphonse Daudet - Textes établis, présentés et annotés. Paris : Gallimard, 3 vol.

\section{RÉSUMÉS}

L'étude de l'œuvre d'Alphonse Daudet nous permettra de constater que les Hommes de Lettres lisaient les œuvres les uns des autres, qu'ils écrivaient à propos de ces œuvres et qu'ils partageaient des espaces de création et de production littéraires. L'écriture et la lecture, des activités largement évoquées dans tous les genres littéraires adoptés par Daudet, sont un lieu commun de sa production littéraire et l'évidence même de son modus vivendi. 
The study of Alphonse Daudet's work allows us to verify that authors used to read each other's work; they wrote about such work and shared moments of creation and literary production activities, vastly referred to in all the authors' adopted literary styles, reflect a common place in his literary production as well as showing a modus vivendi.

INDEX

Mots-clés : lecture, citation, bibliothèque, salon de lecture, éditeurs, journaux, réception Keywords : reading, quotation, library, reception study, editor, newspapers

\section{AUTEUR \\ LÚCIA BANDEIRA}

Agrupamento de Escolas de Esmoriz Ovar Norte luxbandeira[at]gmail.com 